\title{
Quantum theory of the low-frequency linear susceptibility of interferometer-type superconducting qubits
}

\author{
Ya. S. Greenberg ${ }^{1}$ and E. Il'ichev ${ }^{2}$ \\ ${ }^{1}$ Novosibirsk State Technical University, 20 Karl Marx Avenue, 630092 Novosibirsk, Russia \\ ${ }^{2}$ Institut für Photonische Technologien, Jena, Germany
}

(Dated: September 30, 2021)

\begin{abstract}
We use the density matrix formalism to analyze the interaction of interferometer-type superconducting qubits with a high quality tank circuit, which frequency is well below the gap frequency of a qubit. We start with the ground state characterization of the superconducting flux and charge qubits. Then, by making use of a dressed state approach we describe the qubits' spectroscopy when the qubit is irradiated by a microwave field which is tuned to the gap frequency. The last section of the paper is devoted to continuous monitoring of qubit states by using a DC SQUID in the inductive mode.
\end{abstract}

PACS numbers: 74.50.+r, 84.37.+q, resistance, capacitance, inductance, impedance, and admittance, etc.) 03.67.-a

\section{INTRODUCTION}

The existence of a superposition between macroscopically distinct quantum states has important implications, in particular, for the quantum measurement theory, since a single macroscopic quantum system can be easily accessed by the macroscopic measuring device. One of the most advanced solid state quantum systems is a superconducting qubit which is based on either the charge or phase degrees of freedom 1,2 . Several ways of reading out the qubit properties have been proposed and implemented. In an initial research stage switching current measurements combined with the excitation of a qubit by microwave pulses were used ${ }^{3,4}$. Recently it turns out that in some cases inductive (dispersive) measurements can be more effective. In the frame of this approach the qubit is coupled with linear $5,7,8,9,10,11,12,13,14$ or nonlinear oscillators $15,16,17$.

In this paper we study two kinds of interferometer type superconducting qubits (flux and charge qubits) which are realized in practice as Josephson junctions embedded in a superconducting (interferometric) loop $3,10,12$.

A distinct feature of our approach is that a qubit is inductively coupled with a high quality tank circuit (a linear $L_{T} C_{T}$ oscillator, where $L_{T}$ is the inductance and $C_{T}$ is the capacitance of the tank) which resonance frequency is well below the gap frequency of the qubit. The essential information about the qubit properties can be extracted from the voltage across the tank. The method is found to be a reliable technique for the investigation of the properties of the flux qubit systems $\frac{18,19,20,21}{}$, (prior to 2004 see the review paper ${ }^{22}$ and references therein).

Our approach here is based on the rigorous quantum mechanical formulation of the problem, which allows us to apply it to the ground state characterization not only of the flux qubit $\frac{5}{5}$, but of the charge qubit as well. In addition, we show in Section 3 that our method is extremely useful for the investigation of the spectroscopic properties of superconducting qubits.

Since the qubit characteristic frequencies are well above the tank resonance we may consider the tank circuit as a classical system while the qubit is treated quantum mechanically. This description has been proposed in Refs. 5, 6, and the present work is its natural generalization and continuation 23 .

Therefore, the voltage, $V$, across the tank obeys the equation:

$$
\ddot{V}+\gamma_{T} \dot{V}+\omega_{T}^{2} V=-M \omega_{T}^{2} \frac{d\left\langle\widehat{I}_{q}\right\rangle}{d t}+\omega_{T}^{2} L_{T} \dot{I}_{b}
$$

where $\gamma_{T}=\omega_{T} / Q_{T}, Q_{T}$ is the quality factor of the unloaded tank, $M$ is the mutual inductance between the tank and the qubit loop, $\omega_{T}=\left(L_{T} C_{T}\right)^{-1 / 2}$ is the tank resonance frequency, and $I_{b}(t)=I_{0} \cos \omega t$ is the tank bias current with bias frequency $\omega$. Here $\left\langle\widehat{I}_{q}\right\rangle$ is the quantum mechanical average of the quantum operator of the current $\widehat{I_{q}}$ in the interferometer loop.

Eq. (11) in Fourier components reads:

$V(\omega)\left(\omega_{T}^{2}-\omega^{2}+i \omega \gamma_{T}\right)=-M \omega_{T}^{2}\left(\frac{d\left\langle\widehat{I}_{q}\right\rangle}{d t}\right)_{\omega}+i \omega \omega_{T}^{2} L_{T} I_{0}$.

In what follows we assume that the interaction between the qubit and the tank is weak. In this case it would be reasonable to assume (below we prove this assumption by detailed calculations) that

$$
\left(\frac{d\left\langle\widehat{I}_{q}\right\rangle}{d t}\right)_{\omega}=M I_{T}(\omega) Z(\omega)
$$

where $Z(\omega)$ is a complex function $\left(Z(\omega)=Z_{1}(\omega)+\right.$ $\left.i Z_{2}(\omega)\right)$ which depends on the qubit properties only. As is seen from (3) the quantity $d\left\langle\widehat{I}_{q}\right\rangle / d t$ is different from zero only due to the interaction of the qubit with the tank.

By taking into account that $I_{T}(\omega)=-i V(\omega) / \omega L_{T}$, we obtain for the tank detuning $\xi$, and friction $\Gamma_{T}$ :

$$
\xi=\omega_{T}^{2}-\omega^{2}+\frac{k^{2} L_{q} \omega_{T}^{2}}{\omega} Z_{2}(\omega)
$$




$$
\Gamma_{T}=\gamma_{T}-\frac{k^{2} L_{q} \omega_{T}^{2}}{\omega^{2}} Z_{1}(\omega)
$$

where $L_{q}$ is the inductance of the qubit loop, $k^{2}=$ $M^{2} / L_{T} L_{q}$ is a coupling constant, which characterizes the inductive interaction between the qubit and the tank.

From (4) and (5) we obtain the voltage amplitude $V_{T}$, and the phase $\alpha: V_{T}=\omega \omega_{T}^{2} L_{T} I_{0} / \sqrt{\xi^{2}+\omega^{2} \Gamma_{T}^{2}}$, and $\tan \alpha=\xi / \omega \Gamma_{T}$.

Below we calculate in a rigorous quantum mechanical way the low frequency linear response of a qubit to a low frequency probe signal. It is necessary to say that our approach is valid if the interaction between the qubit and the tank is weak which allows us to neglect all nonlinear terms caused by the finite amplitude of the low-frequency signal. Therefore, the main aim here is to calculate the quantity $Z(\omega)$ which transfers the qubit properties to the low frequency characteristics of the tank circuit: its detuning (40) and friction (5).

The paper is organized as follows. In Section 2 the general approach to the ground state characterization of qubits is considered. At the end of the section we obtain the expressions for detuning and friction both for the flux and charge qubits. In Section 3 we consider an interferometer type qubit which is inductively coupled to a low frequency resonant circuit and subjected simultaneously to microwave radiation. By making use of the dressed state approach ${ }^{24}$ we calculate the low frequency susceptibilities for charge and flux qubits. The results obtained in this section are applied to explain the recently found phenomenon of the damping and amplification of the output signal by a superconducting flux qubit ${ }^{25}$. The method developed in this paper is applied in Section 4 to a continuous readout of a superconducting qubit by a DC SQUID in the inductive mode.

\section{GROUND STATE CHARACTERIZATION OF QUBITS}

In this section we consider a qubit which is isolated from microwave fields and interacts inductively with a high quality tank circuit. In what follows we assume the tank frequency is well below the qubit gap frequency $\Delta E$ and, in addition, the temperature is sufficiently low, so that $k_{B} T<<\Delta E$. Under these conditions the signal detected at the output of the tank circuit is mainly defined by the properties of the qubit ground state.

\section{A. Rate equation for two level system}

In the basis of eigenstates $|1\rangle,|2\rangle$ the Hamiltonian of a qubit reads:

$$
H_{0}=\frac{\Delta E}{2} \sigma_{Z}
$$

where $\Delta E$ is the gap between two energy states. The eigenstates are denoted in the following as $|1\rangle$ (ground state) and $|2\rangle$ (excited state) with the properties: $\sigma_{Z}|1\rangle=-|1\rangle, \sigma_{Z}|2\rangle=|2\rangle, \sigma_{X}|1\rangle=|2\rangle, \sigma_{X}|2\rangle=|1\rangle$. $\sigma_{Y}|1\rangle=i|2\rangle, \sigma_{Y}|2\rangle=-i|1\rangle$.

Next we introduce the density matrix:

$$
\frac{d \sigma}{d t}=-\frac{i}{\hbar}\left[H_{0}, \sigma\right]
$$

and define its matrix elements as $\rho_{11}=\langle 1|\sigma| 1\rangle, \rho_{22}=$ $\langle 2|\sigma| 2\rangle, \rho_{12}=\langle 1|\sigma| 2\rangle, \rho_{21}=\langle 2|\sigma| 1\rangle=\rho_{12}^{+}$. From (7) we find the equations for the elements of the density matrix:

$$
\begin{gathered}
\frac{d \rho_{11}}{d t}=0, \\
\frac{d \rho_{22}}{d t}=0, \\
\frac{d \rho_{12}}{d t}=-i \Omega \rho_{12},
\end{gathered}
$$

where $\Omega=\Delta E / \hbar$. In the case of damping the above equations should be changed to:

$$
\begin{gathered}
\frac{d \rho_{11}}{d t}=-\Gamma_{\uparrow} \rho_{11}+\Gamma_{\downarrow} \rho_{22}, \\
\frac{d \rho_{22}}{d t}=\Gamma_{\uparrow} \rho_{11}-\Gamma_{\downarrow} \rho_{22}, \\
\frac{d \rho_{12}}{d t}=-i \Omega \rho_{12}-\Gamma_{\varphi} \rho_{12},
\end{gathered}
$$

where $\Gamma_{\downarrow}$ is the transition rate from state $|2\rangle$ to state 1) (relaxation rate), $\Gamma_{\uparrow}$ is the transition rate from state $|1\rangle$ to state $|2\rangle$ (excitation rate), and the quantity $\Gamma_{\varphi}$ is the rate of decoherence. For equilibrium conditions the relaxation and excitation rates are related by the detailed balance law:

$$
\Gamma_{\uparrow}=\Gamma_{\downarrow} \exp \left(-\frac{\Delta E}{k_{B} T}\right) .
$$

From (14) we obtain

$$
\Gamma_{-} T_{1} \equiv-\rho^{(e q)}=-\tanh \left(\frac{\Delta E}{2 k_{B} T}\right),
$$

where we define $\Gamma_{-}=\Gamma_{\uparrow}-\Gamma_{\downarrow}$ and the longitudinal relaxation time $T_{1}^{-1}=\Gamma_{\uparrow}+\Gamma_{\downarrow}$.

We rewrite Eqs. (11), (12), (13) in operator form

$$
\frac{d \sigma}{d t}=-\frac{i}{\hbar}\left[H_{0}, \sigma\right]+\widehat{L},
$$

where

$$
\begin{aligned}
\widehat{L}=-\Gamma_{\uparrow}|1\rangle\langle 1|\sigma| 1\rangle\left\langle 1\left|+\Gamma_{\downarrow}\right| 1\right\rangle\langle 2|\sigma| 2\rangle\langle 1| \\
+\Gamma_{\uparrow}|2\rangle\langle 1|\sigma| 1\rangle\left\langle 2\left|-\Gamma_{\downarrow}\right| 2\right\rangle\langle 2|\sigma| 2\rangle\langle 2| \\
\quad-\Gamma_{\varphi}|1\rangle\langle 1|\sigma| 2\rangle\left\langle 2\left|-\Gamma_{\varphi}\right| 2\right\rangle\langle 2|\sigma| 1\rangle\langle 1| .
\end{aligned}
$$


As it is seen from (11) and (12), the total population is constant: $\frac{d}{d t}\left(\rho_{11}+\rho_{22}\right)=0$. We take the normalization condition as $\rho_{11}+\rho_{22}=1$. The rate equations can be further simplified by introducing new variables: $\rho=\rho_{11}-$ $\rho_{22}$, which is the difference in populations between the lower and the higher levels, and $\rho_{+}=\rho_{12}+\rho_{21}, \rho_{-}=$ $\rho_{12}-\rho_{21}$ :

$$
\begin{gathered}
\frac{d \rho}{d t}=-\frac{1}{T_{1}} \rho-\Gamma_{-}, \\
\frac{d \rho_{-}}{d t}=-i \Omega \rho_{+}-\Gamma_{\varphi} \rho_{-}, \\
\frac{d \rho_{+}}{d t}=-i \Omega \rho_{-}-\Gamma_{\varphi} \rho_{+} .
\end{gathered}
$$

The quantity $\rho^{(e q)}$ in $(15)$ is just a steady state solution of the equation (18), and is the difference in equilibrium populations between the lower and the higher levels.

\section{B. Interaction between qubit and tank circuit}

The Hamiltonian of a qubit which is coupled to the tank in the eigenbasis reads:

$$
H=\frac{\Delta E\left(\Phi_{X}\right)}{2} \sigma_{Z}+M I_{T} \widehat{I}_{q}
$$

where $I_{T}$ is the current in the tank inductance, $\Phi_{X}$ is a DC bias flux through a qubit loop.

The current operator of the qubit, $\widehat{I}_{q}$, can generally be written as:

$$
\widehat{I}_{q}=I_{X} \sigma_{X}+I_{Y} \sigma_{Y}+I_{Z} \sigma_{Z}
$$

where the quantities $I_{X}, I_{Y}, I_{Z}$, which will be specified below, depend on the nature of the qubit.

The interaction with the tank also influences the $\Gamma$ rates in Eqs. (11), (12). Therefore, we may write in linear approximation

$$
\begin{aligned}
& \Gamma_{\uparrow}^{(\lambda)}=\Gamma_{\uparrow}+\lambda \frac{d \Gamma_{\uparrow}}{d \phi_{X}}, \\
& \Gamma_{\downarrow}^{(\lambda)}=\Gamma_{\downarrow}+\lambda \frac{d \Gamma_{\downarrow}}{d \phi_{X}},
\end{aligned}
$$

where $\lambda=2 \pi M I_{T} / \Phi_{0}, \phi_{X}=2 \pi \Phi_{X} / \Phi_{0}, \Phi_{0}=h / 2 e$ is the flux quantum.

As for $\Gamma_{\varphi}$, we assume this rate is sufficiently high which allows us to neglect its modification by slow time dependent external flux $M I_{T}$.

The equation for the density matrix is similar to (16)

$$
\frac{d \sigma}{d t}=-\frac{i}{\hbar}[H, \sigma]+\widehat{L}
$$

where $H$ is given in (21).

From (25) we get the following equations for the elements of the density matrix:

$\frac{d \rho}{d t}=\frac{2 i \lambda I_{X}}{\hbar} \frac{\Phi_{0}}{2 \pi} \rho_{-}-\frac{2 \lambda I_{Y}}{\hbar} \frac{\Phi_{0}}{2 \pi} \rho_{+}-\frac{1}{T_{1}} \rho-\Gamma_{-}+\lambda \frac{1}{T_{1}} \frac{d \rho^{(e q)}}{d \phi_{X}}$,

$\frac{d \rho_{-}}{d t}=i \Omega \rho_{+}-\Gamma_{\varphi} \rho_{-}+\frac{2 i \lambda I_{Z}}{\hbar} \frac{\Phi_{0}}{2 \pi} \rho_{+}+\frac{2 i \lambda I_{X}}{\hbar} \frac{\Phi_{0}}{2 \pi} \rho$,

$\frac{d \rho_{+}}{d t}=i \Omega \rho_{-}-\Gamma_{\varphi} \rho_{+}+\frac{2 i \lambda I_{Z}}{\hbar} \frac{\Phi_{0}}{2 \pi} \rho_{-}+\frac{2 \lambda I_{Y}}{\hbar} \frac{\Phi_{0}}{2 \pi} \rho$.

\section{Linear susceptibilities for the qubit}

We find the solution to Eqs. (26) - 27), by assuming the coupling $\lambda$ is small. Therefore, the time dependent solution for these equations can be obtained by the perturbation method as the small time dependent corrections to the steady state values: $\rho(t)=\rho^{(0)}+\rho^{(1)}(t)$, $\rho_{+}(t)=\rho_{+}^{(0)}+\rho_{+}^{(1)}(t), \rho_{-}(t)=\rho_{-}^{(0)}+\rho_{-}^{(1)}(t)$, where $\rho^{(0)}$, $\rho_{+}^{(0)}$, and $\rho_{-}^{(0)}$ are steady state solutions for Eqs. (26) (27) with $\lambda=0$. For these steady state values we readily obtain: $\rho^{(0)}=\rho^{(e q)}, \rho_{+}^{(0)}=0, \rho_{-}^{(0)}=0$. Therefore, the fist order corrections $\rho^{(1)}(t), \rho_{+}^{(1)}(t), \rho_{-}^{(1)}(t)$ satisfy the following equations (below we omit superscript 1 ):

$$
\begin{gathered}
\frac{d \rho}{d t}=-\frac{1}{T_{1}} \rho+\lambda \frac{1}{T_{1}} \frac{d \rho^{(e q)}}{d \phi_{X}}, \\
\frac{d \rho_{-}}{d t}=i \Omega \rho_{+}-\Gamma_{\varphi} \rho_{-}+\frac{2 i \lambda I_{X}}{\hbar} \frac{\Phi_{0}}{2 \pi} \rho^{(e q)}, \\
\frac{d \rho_{+}}{d t}=i \Omega \rho_{-}-\Gamma_{\varphi} \rho_{+}+\frac{2 \lambda I_{Y}}{\hbar} \frac{\Phi_{0}}{2 \pi} \rho^{(e q)} .
\end{gathered}
$$

From these equations it is not difficult to find the linear susceptibilities of the system $\left(\chi_{\rho}(\omega)=\rho(\omega) / \lambda(\omega)\right.$, etc., where $\left.\lambda(\omega)=2 \pi M I_{T}(\omega) / \Phi_{0}\right)$. Therefore, we get

$$
\begin{gathered}
\chi_{\rho}(\omega)=\frac{1}{\left(i \omega T_{1}+1\right)} \frac{d \rho^{(e q)}}{d \phi_{X}}, \\
\chi_{\rho_{-}}(\omega)=\frac{2 i \Phi_{0} \rho^{(e q)}}{2 \pi \hbar d(\omega)}\left[\left(i \omega+\Gamma_{\varphi}\right) I_{X}+\Omega I_{Y}\right], \\
\chi_{\rho_{+}}(\omega)=\frac{2 \Phi_{0} \rho^{(e q)}}{2 \pi \hbar d(\omega)}\left[\left(i \omega+\Gamma_{\varphi}\right) I_{Y}-\Omega I_{X}\right],
\end{gathered}
$$

where

$$
d(\omega)=\left(i \omega+\Gamma_{\varphi}\right)^{2}+\Omega^{2}
$$




\section{Calculation of $Z_{1}(\omega)$ and $Z_{2}(\omega)$}

First we calculate the average current $\left\langle\widehat{I}_{q}\right\rangle$. By using (22) we obtain:

$$
\left\langle\widehat{I}_{q}\right\rangle=I_{X} \operatorname{Tr}\left(\sigma \sigma_{X}\right)+I_{Y} \operatorname{Tr}\left(\sigma \sigma_{Y}\right)+I_{Z} \operatorname{Tr}\left(\sigma \sigma_{Z}\right),
$$

where $\operatorname{Tr}\left(\sigma \sigma_{Z}\right)=-\rho, \operatorname{Tr}\left(\sigma \sigma_{X}\right)=\rho_{+}, \operatorname{Tr}\left(\sigma \sigma_{Y}\right)=i \rho_{-}$. Therefore,

$$
\left\langle\widehat{I}_{q}\right\rangle=-I_{Z} \rho+I_{X} \rho_{+}+i I_{Y} \rho_{-} .
$$

With the aid of equations (26), (27), and (28) we obtain:

$$
\begin{aligned}
\frac{d\left\langle\widehat{I}_{q}\right\rangle}{d t} & =\frac{I_{Z}}{T_{1}}\left(\rho+\Gamma_{-} T_{1}-\lambda \frac{d \rho^{(e q)}}{d \phi_{X}}\right) \\
& +i\left(I_{X} \Omega-I_{Y} \Gamma_{\varphi}\right) \rho_{-}-\left(I_{X} \Gamma_{\varphi}+I_{Y} \Omega\right) \rho_{+} .
\end{aligned}
$$

If the interaction between the qubit and the tank is absent $(\lambda=0)$, then, $\rho^{(0)}=\rho^{(e q)}, \rho_{+}^{(0)}=0, \rho_{-}^{(0)}=0$, and, as is seen from (37) and (38), the average current is proportional to the difference of equilibrium populations between the two states: $\left\langle\widehat{I}_{q}\right\rangle=-I_{Z} \rho^{(e q)}$, but $d\left\langle\widehat{I}_{q}\right\rangle / d t=0$. However, if the interaction is sufficiently weak, such that $\lambda \ll 1$, the first approximation for the latter quantity gives:

$$
\begin{aligned}
& \frac{d\left\langle\widehat{I}_{q}\right\rangle}{d t}=\frac{I_{Z}}{T_{1}}\left(\rho^{(1)}-\lambda \frac{d \rho^{(e q)}}{d \phi_{X}}\right) \\
& \quad+i\left(I_{X} \Omega-I_{Y} \Gamma_{\varphi}\right) \rho_{-}^{(1)}-\left(I_{X} \Gamma_{\varphi}+I_{Y} \Omega\right) \rho_{+}^{(1)}
\end{aligned}
$$

and for its Fourier component:

$$
\begin{array}{r}
\left(\frac{d\left\langle\widehat{I}_{q}\right\rangle}{d t}\right)_{\omega}=\frac{I_{Z}}{T_{1}}\left(\chi_{\rho}(\omega)-\frac{d \rho^{(e q)}}{d \phi_{X}}\right) \frac{2 \pi M}{\Phi_{0}} I_{T}(\omega) \\
+\frac{2 \pi M}{\Phi_{0}} I_{T}(\omega)\left[i\left(I_{X} \Omega-I_{Y} \Gamma_{\varphi}\right) \chi_{\rho_{-}}(\omega)\right. \\
\left.-\left(I_{X} \Gamma_{\varphi}+I_{Y} \Omega\right) \chi_{\rho_{+}}(\omega)\right] .
\end{array}
$$

Therefore, we obtain for $Z_{1}(\omega)$ and $Z_{2}(\omega)$ defined in (3):

$$
\begin{gathered}
Z_{1}(\omega)=\frac{2 \pi}{\Phi_{0}} \frac{I_{Z}}{T_{1}}\left(\chi_{\rho}^{\prime}(\omega)-\frac{d \rho^{(e q)}}{d \phi_{X}}\right) \\
-\frac{2 \pi}{\Phi_{0}}\left(I_{X} \Omega-I_{Y} \Gamma_{\varphi}\right) \chi_{\rho_{-}}^{\prime \prime}(\omega)-\frac{2 \pi}{\Phi_{0}}\left(I_{X} \Gamma_{\varphi}+I_{Y} \Omega\right) \chi_{\rho_{+}}^{\prime}(\omega),
\end{gathered}
$$

$$
\begin{aligned}
& Z_{2}(\omega)=\frac{2 \pi}{\Phi_{0}} \frac{I_{Z}}{T_{1}} \chi_{\rho}^{\prime \prime}(\omega) \\
& +\frac{2 \pi}{\Phi_{0}}\left(I_{X} \Omega-I_{Y} \Gamma_{\varphi}\right) \chi_{\rho_{-}}^{\prime}(\omega)-\frac{2 \pi}{\Phi_{0}}\left(I_{X} \Gamma_{\varphi}+I_{Y} \Omega\right) \chi_{\rho_{+}}^{\prime \prime}(\omega),
\end{aligned}
$$

where $\chi_{\rho}^{\prime}(\omega), \chi_{\rho_{-}}^{\prime}(\omega), \chi_{\rho_{+}}^{\prime}(\omega)$ and $\chi_{\rho}^{\prime \prime}(\omega), \chi_{\rho_{-}}^{\prime \prime}(\omega), \chi_{\rho_{+}}^{\prime \prime}(\omega)$ are, respectively, real and imaginary parts of the corresponding susceptibilities (32) - (34).

If we account for the fact that the gap frequency is large, $\Omega \gg \omega, \Gamma_{\varphi}$, we can simplify the calculations of $Z_{1}(\omega)$ and $Z_{2}(\omega)$ to obtain the tank detuning $\xi$, and friction, $\Gamma_{T}$ in the following form:

$$
\begin{aligned}
\xi=\omega_{T}^{2}-\omega^{2}-I_{Z} & \frac{k^{2} \omega_{T}^{2} L_{q}}{\left(1+\omega^{2} T_{1}^{2}\right)} \frac{d \rho^{(e q)}}{d \Phi_{X}} \\
& -\frac{2 k^{2} L_{q} \omega_{T}^{2}}{\Delta E} \rho^{(e q)}\left(I_{X}^{2}+I_{Y}^{2}\right),
\end{aligned}
$$

$$
\begin{aligned}
\Gamma_{T}=\gamma_{T}+I_{Z} & \frac{k^{2} \omega_{T}^{2} L_{q} T_{1}}{\left(1+\omega^{2} T_{1}^{2}\right)} \frac{d \rho^{(e q)}}{d \Phi_{X}} \\
& +\frac{4 k^{2} L_{q} \hbar^{2} \omega_{T}^{2} \Gamma_{\varphi}}{(\Delta E)^{3}} \rho^{(e q)}\left(I_{X}^{2}+I_{Y}^{2}\right) .
\end{aligned}
$$

The expressions (43), (44) are applicable for any kind of interferometer type superconducting qubit once the components $I_{X}, I_{Y}, I_{Z}$ of the current operator are known.

The terms in right hand sides of (43) and (44) which are proportional to $d \rho^{(e q)} / d \Phi_{X}$ reflect the effect of thermalization which value depends on the interplay between the relaxation rate $T_{1}^{-1}$ and the tank frequency $\omega$.

The quantity $d \rho^{(e q)} / d \Phi_{X}$ can be expressed as:

$$
\frac{d \rho^{(e q)}}{d \Phi_{X}}=-\frac{\cosh ^{-2}\left(\frac{\Delta E}{2 k_{B} T}\right)}{k_{B} T} I_{Z},
$$

where we used the fact that $I_{Z}$ can be written as the derivative of the ground state energy $E_{G}$ over the magnetic flux: $I_{Z}=d E_{G} / d \Phi_{X}$, where $E_{G}=-\Delta E / 2$. Since for a proper qubit operation the condition $\Delta E>>$ $k_{B} T$ is necessary, the quantity $d \rho^{(e q)} / d \Phi_{X}$ scales as $\exp \left(-\frac{\Delta E}{k_{B} T}\right)$. Therefore, no matter what the value $\omega T_{1}$ is, the contribution to the tank response of the terms in (43) and (44) which are proportional to $d \rho^{(e q)} / d \Phi_{X}$ can be neglected as compared with the contribution of the last terms in these expressions.

\section{E. Low frequency response of the flux qubit}

For the flux qubit the energy gap is

$$
\Delta E \equiv \Delta_{\varepsilon}=\sqrt{\Delta^{2}+\varepsilon^{2}}
$$

where $\Delta / 2$ is the tunnelling amplitude between degenerate flux states. The bias $\varepsilon$ is controlled by an external dc flux $\Phi_{X}: \varepsilon=2 \Phi_{0} I_{q} f_{X}$, where $I_{q}$ is the critical current of the flux qubit, $f_{X}=\left(\Phi_{X} / \Phi_{0}-1 / 2\right)$.

The current operator of the flux qubit in the eigenstate basis is:

$$
\widehat{I}_{q}=\frac{I_{q}}{\Delta_{\varepsilon}}\left(\varepsilon \sigma_{Z}-\Delta \sigma_{X}\right) .
$$


Therefore, the components of the current operator for a flux qubit are as follows:

$$
I_{X}=-I_{q} \Delta / \Delta_{\varepsilon}, I_{Z}=I_{q} \varepsilon / \Delta_{\varepsilon}, I_{Y}=0 .
$$

From (43) and (44) we obtain the following expressions for the frequency detuning and the friction of the flux qubit

$$
\begin{gathered}
\xi=\omega_{T}^{2}-\omega^{2}-\frac{2 k^{2} \omega_{T}^{2} L_{q} I_{q}^{2} \Delta^{2}}{\Delta_{\varepsilon}^{3}} \rho^{(e q)} \\
\Gamma_{T}=\gamma_{T}+\frac{4 k^{2} L_{q} I_{q}^{2} \Delta^{2}}{\Delta_{\varepsilon}^{3}}\left(\frac{\hbar \omega_{T}}{\Delta_{\varepsilon}}\right)^{2} \Gamma_{\varphi} \rho^{(e q)},
\end{gathered}
$$

where we neglected the terms which are proportional to $d \rho^{(e q)} / d \Phi_{X}$.

The last term in right hand side of (48), which gives the main contribution of the flux qubit to the tank detuning, coincides with the result obtained earlier theoretically ${ }^{5}$ and confirmed by experiment 7 .

The last term in (48) can be expressed in terms of the curvature of the ground state ${ }^{5,7}$.

$$
-\frac{2 k^{2} \omega_{T}^{2} L_{q} I_{q}^{2} \Delta^{2}}{\Delta_{\varepsilon}^{3}} \rho^{(e q)}=k^{2} \omega_{T}^{2} L_{q} \frac{d^{2} E_{G}}{d \Phi_{X}^{2}} \rho^{(e q)} .
$$

This property is a direct consequence of the relation between current components (47)

$$
\frac{d I_{Z}}{d \Phi_{X}}=2 I_{X}^{2}
$$

which seems to be peculiar for the flux qubit only. For other types of superconducting qubits there is no simple relation between the tank detuning and the curvature of the ground state.

As is seen from (49), our theory predicts the modification of the tank quality factor due to the interaction with the qubit. In principle, this effect allows us to measure the dephasing rate $\Gamma_{\varphi}$. However, for the parameters of the flux qubit used in Ref. 7 this effect was rather weak to be experimentally detected.

\section{F. Low frequency response of the charge qubit}

For a charge qubit with different critical currents, $I_{C 1}$ and $I_{C 2}$ of its Josephson junctions the energy gap is

$$
\Delta E=\sqrt{\epsilon_{J}^{2}+C^{2}}
$$

where $\epsilon_{J}^{2}=E_{J 1}^{2}+E_{J 2}^{2}+2 E_{J 1} E_{J 2} \cos \phi_{X}, E_{J 1}=$ $\Phi_{0} I_{C 1} / 2 \pi, E_{J 2}=\Phi_{0} I_{C 2} / 2 \pi, C=4 E_{C}\left(1-n_{g}\right)$. Here the polarization charge on the island $n_{g}$ is controlled by the gate voltage $V_{g}$ via the capacitance $C_{g}$, namely $n_{g}=C_{g} V_{g} / e$.
The components of the current operator are as follows 26 :

$$
\begin{gathered}
I_{Z}=\frac{2 \pi}{\Phi_{0}} \frac{E_{J 1} E_{J 2}}{2 \Delta E} \sin \phi_{X}, \\
I_{X}=\frac{2 \pi}{\Phi_{0}} \frac{E_{J 1}+E_{J 2}}{4 \epsilon_{J}^{2}} \sin \frac{\phi_{X}}{2} \\
\times\left[\left(E_{J 1}-E_{J 2}\right)^{2}-\frac{4 C E_{J 1} E_{J 2}}{\Delta E} \cos ^{2} \frac{\phi_{X}}{2}\right], \\
I_{Y}=\frac{2 \pi}{\Phi_{0}} \frac{E_{J 1}-E_{J 2}}{4 \epsilon_{J}^{2}} \cos \frac{\phi_{X}}{2} \\
\times\left[\left(E_{J 1}+E_{J 2}\right)^{2}+\frac{4 C E_{J 1} E_{J 2}}{\Delta E} \sin ^{2} \frac{\phi_{X}}{2}\right] .
\end{gathered}
$$

In case of symmetrical junctions $\left(E_{J 1}=E_{J 2}=E_{J}\right)$ we have: $I_{Y}=0$,

$$
\begin{gathered}
I_{Z}=\frac{\pi E_{J}}{\Phi_{0} \Delta E} \sin \phi_{X}, \\
I_{X}=-\frac{\pi E_{J} C}{\Phi_{0} \Delta E} \sin \frac{\phi_{X}}{2},
\end{gathered}
$$

where

$$
\Delta E=\sqrt{4 E_{J}^{2} \cos ^{2} \frac{\phi_{X}}{2}+C^{2}} .
$$

For the tank detuning and friction we obtain from (43) and (44), neglecting the terms which are proportional to $d \rho^{(e q)} / d \Phi_{X}$ :

$$
\begin{gathered}
\xi=\omega_{T}^{2}-\omega^{2}-\frac{2 k^{2} L_{q} \omega_{T}^{2}}{\Delta E} \rho^{(e q)}\left(I_{X}^{2}+I_{Y}^{2}\right), \\
\Gamma_{T}=\gamma_{T}+\frac{4 k^{2} L_{q} \hbar^{2} \omega_{T}^{2} \Gamma_{\varphi}}{(\Delta E)^{3}} \rho^{(e q)}\left(I_{X}^{2}+I_{Y}^{2}\right) .
\end{gathered}
$$

As distinct from the flux qubit, the tank detuning caused by the charge qubit cannot, in general, be expressed in terms of the ground state curvature. It can be done only for the symmetrical case $\left(E_{J 1}=E_{J 2}\right)$ at the point $\phi_{X}=$ $\pi$.

From expression (58) we calculate the dependence of the phase shift $\alpha$ of the output signal on the gate charge parameter $n_{g}$ for different values of magnetic flux $\Phi_{X} / \Phi_{0}$ applied to the qubit loop (see Fig. 11). For the calculations we take the following values for the tank, $Q_{T}=1000$, $k^{2}=0.01, \omega_{T}=2 \pi \times 50 \mathrm{MHz}$, and for the charge qubit, $L_{q}=1.5 \mathrm{nH}, E_{J 1} / h=25 \mathrm{GHz}, E_{J 2} / h=29 \mathrm{GHz}$, $E_{C} / h=3.5 \mathrm{GHz}$.

In conclusion to this section it is worth noting that the average current in the qubit loop $\left\langle\widehat{I}_{q}\right\rangle$ is proportional to the difference of equilibrium populations between the two qubit states only in the absence of interaction between the qubit and the tank circuit: $\left\langle\widehat{I}_{q}\right\rangle=-I_{Z} \rho^{(e q)}$. The other components of the current, $I_{X}$ and $I_{Y}$ which give the main contribution to the tank response (the last terms in (43) and (44)), appear in the average current only due to the interaction of the qubit with the tank. 


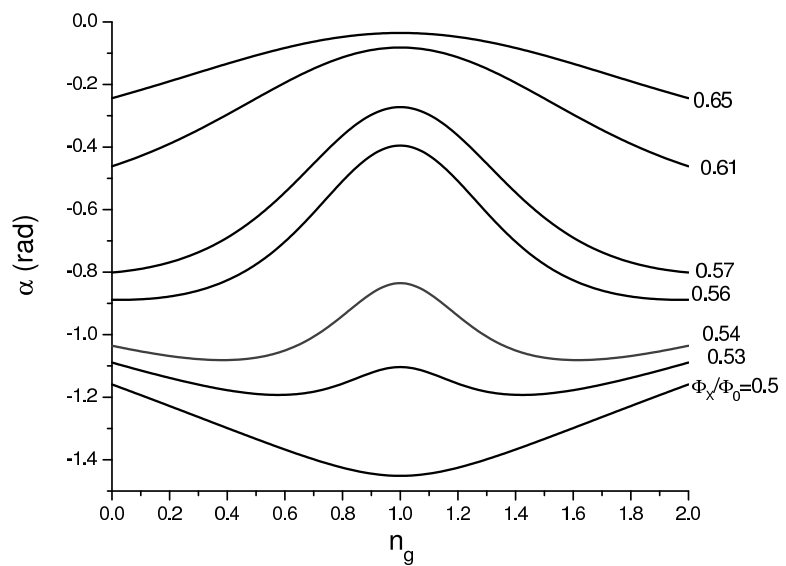

FIG. 1: The dependence of the phase shift $\alpha$ on the gate parameter $n_{g}$ for different values of magnetic flux $\Phi_{X} / \Phi_{0}$ applied to the qubit loop.

\section{CHARACTERIZATION OF IRRADIATED QUBIT BY RABI SPECTROSCOPY}

The essence of the Rabi spectroscopy method is the following. Under microwave irradiation, which frequency is close to the gap frequency, the level structure of the global system (qubit, radiation field and their interaction) is a ladder of pairs of states where the spacing between two levels in the pair is equal to the Rabi frequency, the value of which is controlled by the power of microwaves 24 . Normally, Rabi oscillations are damped out with a rate, which depends on how strongly the system is coupled to the environment. However, if a second low-frequency source (in our case this source is a low frequency excitation from the tank) is applied simultaneously to the qubit it responds with persistent low frequency oscillations. The amplitude of these lowfrequency oscillations has a resonance at the Rabi frequency and its width is dependent on the damping rates of the system 27,28 . Therefore, the interaction of the qubit with the tank induces the transitions between the Rabi levels which result in low frequency oscillations of the current in a qubit loop which, in turn, result in the measurable response of the $\operatorname{tank}^{29}$. In particular, the signature of a high frequency resonance can be read out from the low frequency voltage-flux characteristic of the tank circuit.

Below we assume that the interaction between the qubit and microwave field does not influence the dephasing and the relaxation rates. As was shown in Refs. 30 and 31 , this assumption is valid for relative weak driving, sufficient short correlation time of the heat bath $\tau_{c}$, and in the large temperature limit: $F<<\Delta_{\varepsilon}, \hbar / \tau_{c}, k_{B} T^{32}$.

\section{A. Low frequency linear susceptibility of an irradiated qubit coupled to a tank circuit}

In this subsection we summarize in a concise form the main results from Ref. 29, which are relevant for specific qubit applications.

The Hamiltonian for an irradiated qubit which interacts with a low frequency tank circuit is as follows:

$$
H=\frac{\Delta E}{2} \sigma_{Z}+\hbar \omega_{0} a^{+} a+H_{\mathrm{int}}+H_{\mathrm{int}}^{\mathrm{LF}},
$$

where the second term in (60) describes a microwave field, the third term describes the interaction of the qubit with this microwave field:

$$
H_{\text {int }}=-\frac{1}{2} \widehat{I}_{q} F\left(a^{+}+a\right),
$$

where $\widehat{I}_{q}$ is the current operator of the qubit as given in (22), $F$ is the amplitude of the microwave field with the dimension of a magnetic flux. The last term in (60) describes the interaction of the qubit with a tank circuit:

$$
H_{\mathrm{int}}^{\mathrm{LF}}=M I_{T} \widehat{I}_{q}=M I_{T}\left(I_{Z} \sigma_{Z}+I_{X} \sigma_{X}+I_{Y} \sigma_{Y}\right) .
$$

We denote the eigenfunctions of the photon field as $|N\rangle: a^{+}|N\rangle=\sqrt{N+1}|N+1\rangle$, and $a|N\rangle=\sqrt{N} \mid N-$ $1\rangle$. The eigenfunctions of the noninteracting qubit and associated photon system we take in the form of a tensor product $|1, N\rangle \equiv|1\rangle \otimes|N\rangle$, and $|2, N\rangle \equiv|2\rangle \otimes|N\rangle$.

If the photon frequency $\omega_{0}$ is close to the qubit gap frequency $\Delta E / \hbar$, and the high frequency detuning $\delta$ is small, $\delta=\omega_{0}-\Delta E / \hbar \ll \omega_{0}, \Delta E / \hbar$, where for definitiveness we assume $\delta>0$, then the energies of the states $|1, N+1\rangle$ and $|2, N\rangle$ are close to each other: $E_{1, N+1}-E_{2, N}=\hbar \delta$. The same is true for the pairs of states $|1, N\rangle$ and $|2, N-1\rangle,|1, N+2\rangle$ and $|2, N+1\rangle$, and so on. Therefore, the energy levels of the system under consideration are a ladder of pairs of states which are specified by the photon number $N$. The spacing between two levels in the pair is equal to $\hbar \delta$, and the distance between neighboring pairs is equal to the photon energy $\hbar \omega_{0}{ }^{24}$. These levels of uncoupled qubit-photon system are modified due to the interaction (61). This interaction results in an increase of the energy gap between two levels in the pair. The spacing between the states $|1, N\rangle$ and $|2, N-1\rangle$ becomes equal to $\hbar \Omega_{R}$, where $\Omega_{R}$ is the Rabi frequency 33

$$
\Omega_{R}=\sqrt{\delta^{2}+\Omega_{1}^{2}}
$$

where $\hbar \Omega_{1}=F \sqrt{I_{X}^{2}+I_{Y}^{2}}$.

From now on we will call these two nearby dressed states Rabi levels.

As was shown in Ref. 29 the interaction (62) between the qubit and the tank results in the transitions between Rabi levels. It is just these transitions which result in the low frequency response of a qubit detected by the tank. 
In Ref. 29 we obtained the evolution equations for the elements of the density matrix which describe the transitions between these Rabi levels: $\rho, \rho_{+}$, and $\rho_{-}$, where $\rho$ is the difference of the populations between higher and lower Rabi levels. (Note, that here the definition of $\rho$ is different from that given in Section II). These elements of the density matrix are usually accounted for by a so called rotating wave approximation (RWA). The equations for the elements of the density matrix which describe in RWA the interaction of an irradiated qubit with the tank are as follows:

$$
\begin{gathered}
\frac{d \rho}{d t}=-A_{1} \rho+B \rho_{+}+\frac{2 i \lambda \Phi_{0}}{2 \pi \hbar} I_{Z} \sin 2 \theta \rho_{-}+\Gamma_{-} \cos 2 \theta,(64) \\
\frac{d \rho_{+}}{d t}=-i \Omega_{R} \rho_{-}+B \rho-A_{2} \rho_{+}-\frac{2 i \lambda \Phi_{0}}{2 \pi \hbar} I_{Z} \cos 2 \theta \rho_{-}+\Gamma_{-} \sin 2 \theta,
\end{gathered}
$$

$$
\frac{d \rho_{-}}{d t}=-i \Omega_{R} \rho_{+}-\Gamma_{\varphi} \rho_{-}-\frac{2 i \lambda \Phi_{0}}{2 \pi \hbar} I_{Z}\left(\rho_{+} \cos 2 \theta-\rho \sin 2 \theta\right),
$$

$$
\rho_{+}^{(0)}=\frac{\Gamma_{\varphi}^{2}}{\frac{\Gamma_{\varphi}^{2}}{T_{1}}+A_{1} \Omega_{R}^{2}} \Gamma_{-} \sin 2 \theta,
$$

$$
B=\left[\Gamma_{\varphi}-\frac{1}{T_{1}}\right] \sin 2 \theta \cos 2 \theta
$$

The angle $\theta$ is given by $\tan 2 \theta=-\Omega_{1} / \delta$, where $0<2 \theta<$ $\pi$, so that $\cos 2 \theta=-\delta / \Omega_{R}, \cos \theta=\frac{1}{\sqrt{2}}\left(1-\frac{\delta}{\Omega_{R}}\right)^{1 / 2}$, and $\sin \theta=\frac{1}{\sqrt{2}}\left(1+\frac{\delta}{\Omega_{R}}\right)^{1 / 2}$.

The steady state solution for the elements of the density matrix and the low frequency linear susceptibility of a qubit are as follows 29 :

$$
\rho^{(0)}=\frac{\left(\Gamma_{\varphi}^{2}+\Omega_{R}^{2}\right)}{\frac{\Gamma_{\varphi}^{2}}{T_{1}}+A_{1} \Omega_{R}^{2}} \Gamma_{-} \cos 2 \theta,
$$

$$
\begin{aligned}
& A_{1}=\left[\frac{1}{T_{1}} \cos ^{2} 2 \theta+\Gamma_{\varphi} \sin ^{2} 2 \theta\right], \\
& A_{2}=\left[\frac{1}{T_{1}} \sin ^{2} 2 \theta+\Gamma_{\varphi} \cos ^{2} 2 \theta\right],
\end{aligned}
$$

where

$$
D(\omega)=\left(i \omega+\Gamma_{\varphi}\right)^{2}\left(i \omega+\frac{1}{T_{1}}\right)+\left(i \omega+A_{1}\right) \Omega_{R}^{2} .
$$

It is interesting to note that under high frequency irradiation the population of the Rabi levels becomes inverted. It is seen from (70), where the quantity $\rho^{(0)}$, which is, by definition, the difference of the populations between higher and lower Rabi levels, is positive, since for $\delta>0$ we have $\cos 2 \theta=-\delta / \Omega_{R}<0$, and always
$\Gamma_{-}<0$. In addition, as $\delta$ tends to zero, $\rho^{(0)} \rightarrow 0$ which causes the equalization of the population of the two levels $\left(\rho_{11}=\rho_{22}=\frac{1}{2}\right)$ when the high frequency irradiation is in exact resonance with the energy gap of the qubit. 


\section{B. Calculation of $Z_{1}(\omega)$ and $Z_{2}(\omega)$ for irradiated qubit}

Since the matrix elements of $\sigma_{X}$ and $\sigma_{Y}$ between the Rabi levels are zero, the average current is:

$$
\left\langle\widehat{I}_{q}\right\rangle=I_{Z} \operatorname{Tr}\left(\sigma \sigma_{Z}\right)=I_{Z}\left(\rho(t) \cos 2 \theta+\rho_{+}(t) \sin 2 \theta\right) .
$$

By using Eqs. (64) - (66) we obtain:

$$
\begin{aligned}
\frac{d\left\langle\widehat{I}_{q}\right\rangle}{d t}=-\frac{I_{Z}}{T_{1}}[\rho(t) & \cos 2 \theta+\rho_{+}(t) \sin 2 \theta \\
& \left.+i \Omega_{R} T_{1} \sin 2 \theta \rho_{-}(t)\right]+I_{Z} \Gamma_{-} .
\end{aligned}
$$

This equation can be rewritten as follows:

$$
\frac{d\left\langle\widehat{I}_{q}\right\rangle}{d t}=-\frac{1}{T_{1}}\left\langle\widehat{I}_{q}\right\rangle-i I_{Z} \Omega_{R} \sin 2 \theta \rho_{-}(t)+I_{Z} \Gamma_{-} .
$$

With the aid of the steady state solutions (70) - (72) we find the stationary current when the interaction between the qubit and the tank is absent:

$$
\left\langle\widehat{I}_{q}\right\rangle^{s t}=-I_{Z} \rho^{(e q)} \frac{1+\delta^{2} T_{2}^{2}}{1+\delta^{2} T_{2}^{2}+T_{1} T_{2} \Omega_{1}^{2}},
$$

where $T_{2}=1 / \Gamma_{\varphi}$.

It is not unexpected that this expression is quite similar to the one for the longitudinal magnetization in $\mathrm{NMR}^{34}$. However, the important difference is that the frequency $\Omega_{1}$ depends essentially on the current components of the qubit $\left(\Omega_{1}=F \sqrt{I_{X}^{2}+I_{Y}^{2}}\right)$.

For the Fourier component of (78) we obtain:

$$
\begin{aligned}
& \left(\frac{d\left\langle\widehat{I}_{q}\right\rangle}{d t}\right)_{\omega}=-\frac{2 \pi M}{\Phi_{0}} I_{T}(\omega) \frac{I_{Z}}{T_{1}}\left[\chi_{\rho}(\omega) \cos 2 \theta\right. \\
& \left.\quad+\chi_{\rho_{+}}(\omega) \sin 2 \theta+i \Omega_{R} T_{1} \sin 2 \theta \chi_{\rho_{-}}(\omega)\right] .
\end{aligned}
$$

Therefore, for irradiated qubit the quantities $Z_{1}(\omega)$ and $Z_{2}(\omega)$ are as follows:

$$
\begin{aligned}
Z_{1}(\omega)= & -\frac{2 \pi}{\Phi_{0}} \frac{I_{Z}}{T_{1}}\left[\chi_{\rho}^{\prime}(\omega) \cos 2 \theta\right. \\
& \left.+\chi_{\rho_{+}}^{\prime}(\omega) \sin 2 \theta-\Omega_{R} T_{1} \chi_{\rho_{-}}^{\prime \prime}(\omega) \sin 2 \theta\right], \\
Z_{2}(\omega)= & -\frac{2 \pi}{\Phi_{0}} \frac{I_{Z}}{T_{1}}\left[\chi_{\rho}^{\prime \prime}(\omega) \cos 2 \theta\right. \\
& \left.+\chi_{\rho_{+}}^{\prime \prime}(\omega) \sin 2 \theta+\Omega_{R} T_{1} \chi_{\rho_{-}}^{\prime}(\omega) \sin 2 \theta\right] .
\end{aligned}
$$

In this case the tank detuning $\xi$ and the friction $\Gamma_{T}$ can readily be obtained from (4) and (5) by using the expressions (83) and (82) for $Z_{2}(\omega)$ and $Z_{1}(\omega)$, respectively.

$$
\begin{aligned}
\xi= & \omega_{T}^{2}-\omega^{2}+\rho^{(e q)} \frac{2 k^{2} L_{q} I_{Z}^{2} \omega_{T}^{2}}{\omega \hbar \Omega_{R} T_{1}} \frac{\delta \Gamma_{\varphi} \Omega_{1}}{\Gamma_{\varphi}^{2}+\delta^{2}+T_{1} \Gamma_{\varphi} \Omega_{1}^{2}} \\
& \times\left[-f_{\rho}^{\prime \prime}(\omega)+\frac{\Omega_{1}}{\Omega_{R}} f_{\rho_{+}}^{\prime \prime}(\omega)-\frac{\Omega_{R} T_{1} \Omega_{1}}{\Gamma_{\varphi}} f_{\rho_{-}}^{\prime}(\omega)\right],
\end{aligned}
$$

$$
\begin{aligned}
\Gamma_{T} & =\gamma_{T}-\rho^{(e q)} \frac{2 k^{2} L_{q} I_{Z}^{2} \omega_{T}^{2}}{\omega^{2} \hbar \Omega_{R} T_{1}} \frac{\delta \Gamma_{\varphi} \Omega_{1}}{\Gamma_{\varphi}^{2}+\delta^{2}+T_{1} \Gamma_{\varphi} \Omega_{1}^{2}} \\
& \times\left[-f_{\rho}^{\prime}(\omega)+\frac{\Omega_{1}}{\Omega_{R}} f_{\rho_{+}}^{\prime}(\omega)+\frac{\Omega_{R} T_{1} \Omega_{1}}{\Gamma_{\varphi}} f_{\rho_{-}}^{\prime \prime}(\omega)\right],
\end{aligned}
$$

where

$$
\begin{array}{r}
f_{\rho}(\omega)=\frac{1}{D(\omega)}\left[\operatorname { s i n } 2 \theta \left[\left(i \omega+\Gamma_{\varphi}\right)\right.\right. \\
\left.\left(i \omega+\frac{1}{T_{1}}\right)+\Omega_{R}^{2}\right] \\
\left.+\frac{\Omega_{R}^{2}}{\Gamma_{\varphi}} B \cos 2 \theta\right],
\end{array}
$$

$f_{\rho_{+}}(\omega)=\frac{1}{D(\omega)}\left[\left(i \omega+\Gamma_{\varphi}\right)\left(i \omega+\frac{1}{T_{1}}\right)-\left(i \omega+A_{1}\right) \frac{\Omega_{R}^{2}}{\Gamma_{\varphi}}\right]$

$$
f_{\rho_{-}}(\omega)=\frac{i}{D(\omega)}\left(i \omega+\frac{1}{T_{1}}\right)\left(i \omega+2 \Gamma_{\varphi}\right)
$$

The expressions (84) and (85) can equally be used for the flux and the charge qubit with due account for the different current components (47) for the flux qubit and (52) - (54) for the charge qubit), and the different structure of the energy gap (45) for the flux qubit and (51) for the charge qubit).

\section{Damping and amplification by a superconducting flux qubit}

A remarkable property of the irradiated flux qubit coupled to a low frequency $L C$ circuit is that the effective quality factor of the tank can be made several times higher (lower) than that for an unloaded tank. A clear phenomenological explanation of this effect, which has been found experimentally, has been given in Ref. 25, where the effect has also been explained by a rigorous quantum treatment of the problem followed by a numerical solution of the corresponding equations for the global density matrix of the qubit-tank system.

Here we show analytically that in the frame of our approach the effect results from the frequency dependent quality factor of the tank coupled to an irradiated qubit. The analytical expression we obtain for the quality factor is valid for small high frequency detuning, $\delta<<\omega_{0}$, $\Delta E / \hbar$ which allows us to account only for the transitions between the Rabi levels. As is seen from (85) the amplification occurs if the high frequency detuning $\delta$ is positive. It means that on the energy diagram of the qubit the working point of the driving current from the tank is located to the left from the point of the high frequency resonance (see Fig. 1 in Ref. 25). Positive values of $\delta$ correspond to the inverted population of the Rabi levels (see Eq. (70)). It is just the emission of these Rabi photons which pumps the energy into the tank. For negative values of $\delta$ the working point of the driving current from the tank is located to the right from the point of the 


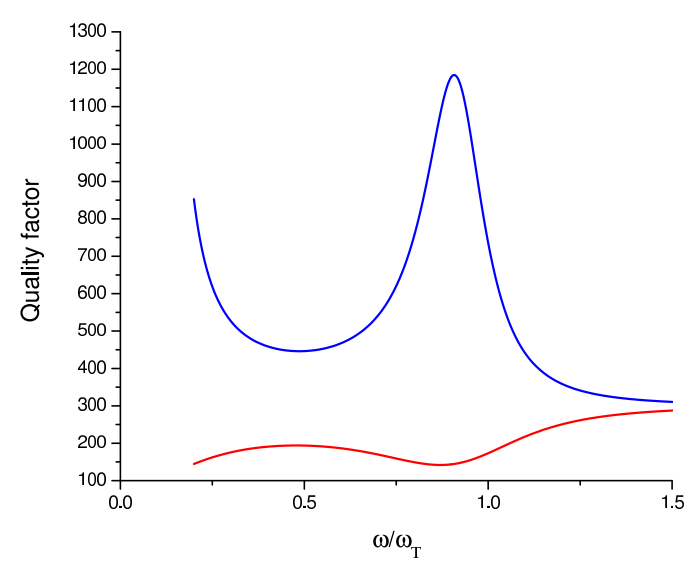

FIG. 2: (Color online). The dependence of the quality factor of the tank coupled to an irradiated qubit. The quality of unloaded tank $Q_{T}=300$. The upper curve $\left(f_{X}=0.0027, \delta>0\right)$ shows the effect of the amplification. The lower curve $\left(f_{X}=\right.$ $0.004, \delta<0)$ demonstrates the effect of the damping.

high frequency resonance. For this case the lower Rabi level is more populated than the higher level, and the tank transfers the energy to the Rabi levels.

From (85) the quality factor of the tank coupled to the qubit can be written as $\frac{1}{Q}=\frac{1}{Q_{T}}+\frac{1}{Q_{q b}}$, where

$$
\begin{aligned}
\frac{1}{Q_{q b}} & =-\rho^{(e q)} \frac{2 k^{2} L_{q} I_{q}^{2} \varepsilon^{2} \omega_{T}}{\Delta_{\varepsilon}^{2} \omega^{2} \hbar \Omega_{R} T_{1}} \frac{\delta \Gamma_{\varphi} \Omega_{1}}{\Gamma_{\varphi}^{2}+\delta^{2}+T_{1} \Gamma_{\varphi} \Omega_{1}^{2}} \\
& \times\left[-f_{\rho}^{\prime}(\omega)+\frac{\Omega_{1}}{\Omega_{R}} f_{\rho_{+}}^{\prime}(\omega)+\frac{\Omega_{R} T_{1} \Omega_{1}}{\Gamma_{\varphi}} f_{\rho_{-}}^{\prime \prime}(\omega)\right] .
\end{aligned}
$$

As is seen from (89), $Q=Q_{T}$ at the point $f_{X}=0(\varepsilon=$ $\left.2 \Phi_{0} I_{q} f_{X}\right)$. The reason for this is the vanishing of the current component $I_{Z}$ at this point (see (47)).

We calculate from expression (85) the frequency dependence of the tank quality factor $Q$ and the voltagefrequency curve for the tank coupled to the irradiated qubit. We take the following parameters of the tank, $Q_{T}=300, k^{2}=0.001, \omega_{T}=2 \pi \times 50 \mathrm{MHz}$, and of the qubit, $L_{q}=25 \mathrm{pH}, I_{q}=300 \mathrm{nA}, \Gamma_{\varphi}=4 \times 10^{7} \mathrm{c}^{-1}$, $T_{1}=1.25 \times 10^{-8} \mathrm{c}, \Delta / h=3.78 \mathrm{GHz}$. For the microwave frequency we take $\omega_{0}=2 \pi \times 3.81 \mathrm{GHz}$, and the microwave power in the frequency units is $F / h=2 \pi \times 45$ $\mathrm{MHz}$.

The dependence of the tank quality factor on the low frequency is shown in Fig. 2.

The dependence of the normalized tank voltage on the low frequency (bias frequency of the tank) calculated for the same data as those for Fig. 2 is shown in Fig. 3 , For the same data we also show in Fig. 4 the dependence of the voltage and the tank quality on the bias DC flux $f_{X}$. The curves are plotted for a tank resonance $\omega=\omega_{T}$. The blue (short) arrows at this figure show two points where the microwave frequency matches exactly the gap frequency $\omega_{0}=\Delta_{\varepsilon} / \hbar$. Between these points the

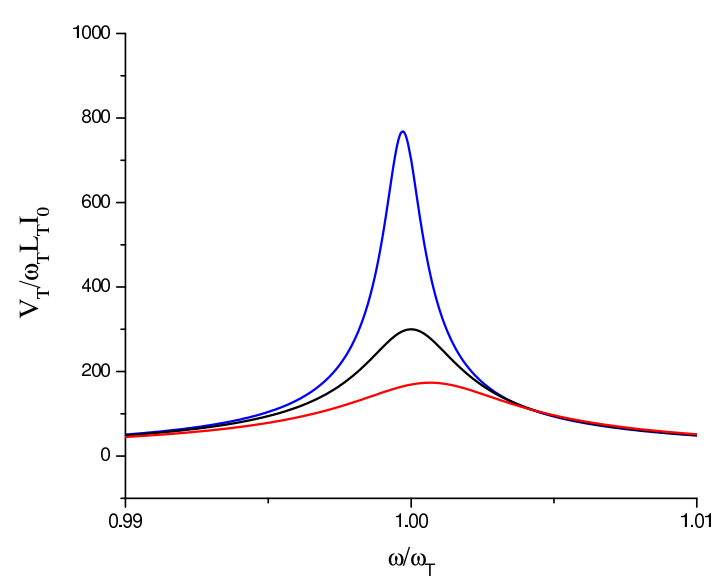

FIG. 3: (Color online). The dependence of the normalized tank voltage on the low frequency. The quality factor of the unloaded tank $Q_{T}=300$. The upper curve $\left(f_{X}=0.0027, \delta>0\right)$ shows the effect of the amplification. The lower curve $\left(f_{X}=0.004, \delta<0\right)$ is the effect of the damping. The curve, which is in between, is the voltage curve for the unloaded tank.

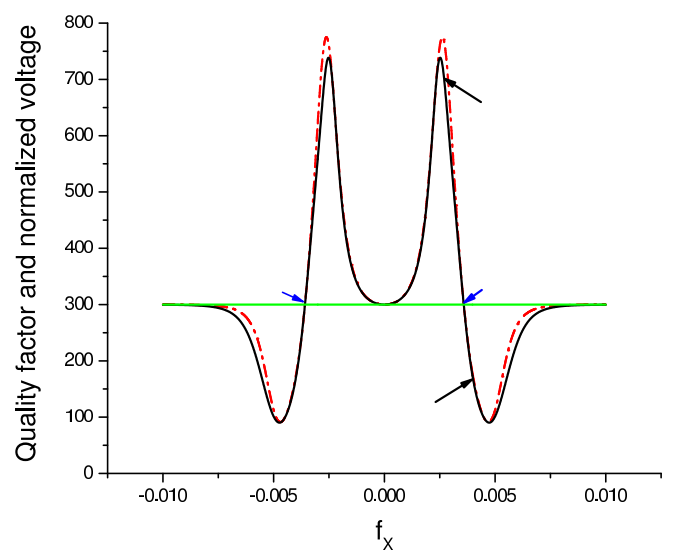

FIG. 4: (Color online). The dependence of the normalized tank voltage (black curve) and the tank quality factor (red dash-dot curve) on the bias flux $f_{X}$, plotted for $\omega=\omega_{T}$. The quality factor of the unloaded tank $\left(Q_{T}=300\right)$ is shown by the green straight line. The black (long) arrows show the points which correspond to the voltage at the tank resonance in Fig. 3. The blue (short) arrows show the points where the microwave frequency matches exactly the gap frequency $\omega_{0}=\Delta_{\varepsilon} / \hbar$.

high frequency detuning $\delta$ is positive, and the Rabi photons pump the energy into the tank. Beyond them the high frequency detuning $\delta$ is negative, and the energy is drained off the tank.

The analysis of Eqs. (84) and (85) shows that the detuning of the tank due to the interaction with the qubit is rather small, therefore, the resonance frequency of the coupled tank is practically equal to the unloaded value $\omega_{T}$. However, the quality factor of the coupled tank has 


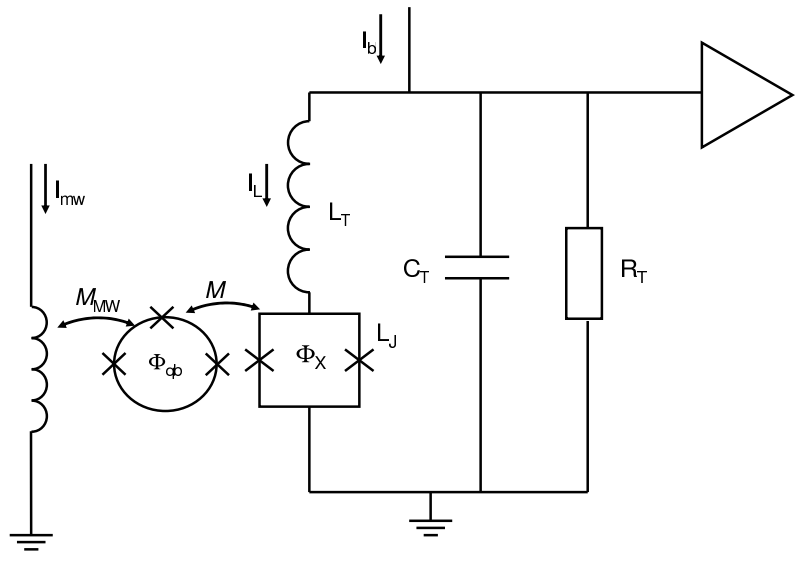

FIG. 5: Flux qubit coupled to the inductive DC SQUID, which is incorporated in a resonant tank circuit.

a strong dependence on the frequency. Generally, the position of the peak value of the quality factor does not coincide with the resonance frequency of the tank (see Fig. 21). Therefore, the tank voltage curve can be appreciably differed from the lorentzian line.

\section{CONTINUOUS READOUT OF A SUPERCONDUCTING QUBIT USING A DC SQUID IN THE INDUCTIVE MODE}

This method has been realized in Refs. 15 and 38 . In these works a DC SQUID operating in the inductive mode (the bias current through the SQUID is less than its critical current) was inductively coupled to a flux qubit. To enhance the measurement sensitivity, DC SQUID was incorporated in a resonant $L C$ circuit (see Fig. 5). The spectroscopic measurements in Refs. 15 and 38 have been performed by measuring the switching currents of the DC SQUID after the microwave pulse had been applied to the qubit.

Here we propose the modification of the method which does not require a measurement of the switching currents. Our method is based on the detection of the response of the resonant $L C$ circuit in which a DC SQUID is incorporated. It is assumed that in the process of the measurement the qubit is irradiated by microwaves continuously. We show that the DC SQUID in the inductive mode directly measures the $I_{Z}$ component of the current in a qubit loop. Our approach can be applied both for flux and charge qubits.

First, we write the well known expression for the current across the DC SQUID

$$
I_{L}=I_{C} \cos \left(f_{s q}\right) \sin \gamma,
$$

where $I_{L}$ is the current in the inductor $L_{T}$, which is connected in series with the SQUID loop, $I_{C}$ is the critical current of the SQUID, $\gamma$ is the Josephson phase, which is related to the voltage across a SQUID by the Josephson expression $V_{s q}=\frac{\Phi_{0}}{2 \pi} \frac{d \gamma}{d t}$. The quantity $f_{s q}$ is the normalized total flux in the SQUID loop, $\Phi_{s q}$, which is the sum of the external control flux $\Phi_{X}$ and the flux $M\left\langle\widehat{I}_{q}\right\rangle$ from the qubit:

$$
f_{s q} \equiv \frac{\pi \Phi_{s q}}{\Phi_{0}}=\frac{\pi \Phi_{X}}{\Phi_{0}}+\frac{\pi M\left\langle\widehat{I}_{q}\right\rangle}{\Phi_{0}} .
$$

In this expression we neglected the flux $M I_{c i r c}$, which is generated in the SQUID loop by a circulating current $I_{\text {circ }}$. Below we assume $I_{L}<<I_{C}$, which implies $\gamma<<1$. From (90) we get $\gamma=I_{L} / I_{C} \cos f_{s q}$, and for the voltage across the tank we have:

$$
V=\left(L_{T}+L_{J}\right) \frac{d I_{L}}{d t}
$$

where

$$
L_{J}=\frac{\Phi_{0}}{2 \pi I_{C} \cos f_{s q}}
$$

is the Josephson inductance of the DC SQUID. The equation for the voltage across the tank is similar to (11):

$$
\ddot{V}+\gamma_{T} \dot{V}+\frac{\omega_{T}^{2}}{1+\frac{L_{J}}{L_{T}}} V=\omega_{T}^{2} L_{T} \dot{I}_{b} .
$$

We assume the coupling of the qubit to the tank is weak: $\frac{\pi M\left\langle\widehat{I}_{q}\right\rangle}{\Phi_{0}}<<1$. For this case Eq. (94) can be written in the following form:

$$
\begin{aligned}
& \ddot{V}+\gamma_{T} \dot{V}+\omega_{T}^{2} \frac{\cos f_{X}}{\cos f_{X}+\frac{L_{J}^{(0)}}{L_{T}}} V \\
& -\omega_{T}^{2} \frac{k}{2}\left(\frac{L_{q}}{L_{T}}\right)^{\frac{1}{2}} \frac{\sin f_{X}}{\left(\cos f_{X}+\frac{L_{J}^{(0)}}{L_{T}}\right)^{2}} \frac{\left\langle\widehat{I}_{q}\right\rangle}{I_{C}} V=\omega_{T}^{2} L_{T} \dot{I}_{b},
\end{aligned}
$$

where $f_{X}=\frac{\pi \Phi_{X}}{\Phi_{0}}, L_{J}^{(0)}=\frac{\Phi_{0}}{2 \pi I_{C}}$.

As it is seen from this expression the tank response is proportional to the averaged qubit current, but not to its time derivative. Therefore, we may take for the quantity $\left\langle\widehat{I}_{q}\right\rangle$ in (95) its stationary time independent part $\left\langle\widehat{I}_{q}\right\rangle^{s t}$. By doing this we may neglect the off diagonal components of the density matrix $\rho_{+}$, and $\rho_{-}$since their stationary parts are proportional to the coupling constant $k$, and their contribution to the tank response scales as $k^{2}$.

Therefore, the influence of the qubit results in the tank detuning

$$
\xi=\omega_{T}^{2}-\omega^{2}-\omega_{T}^{2} \frac{k}{\sqrt{2}}\left(\frac{L_{q}}{L_{T}}\right)^{\frac{1}{2}} \frac{\left\langle\widehat{I}_{q}\right\rangle^{s t}}{I_{C}},
$$

where we take $f_{X}=\pi / 4$ and assume $L_{J}^{(0)}<<L_{T}$. The detuning can be detected from the phase of the output signal at resonance:

$$
\tan \alpha=-\frac{k Q_{T}}{\sqrt{2}}\left(\frac{L_{q}}{L_{T}}\right)^{\frac{1}{2}} \frac{\left\langle\widehat{I}_{q}\right\rangle^{s t}}{I_{C}} .
$$


The expressions (96) and (97)can equally be applied both for ground state and spectroscopic measurements. For the ground state measurements (the microwaves are absent) we have from (37) $\left\langle\widehat{I}_{q}\right\rangle^{s t}=-I_{Z} \rho^{(e q)}$. For spectroscopic measurements of an irradiated qubit $\left\langle\widehat{I}_{q}\right\rangle^{s t}$ is given in (80).

In fact, DC SQUID measures not an absolute value of the flux threading its loop, but the change of the flux. Therefore, we may subtract the ground state measurements from those for an irradiated qubit to obtain a pure lorentzian line

$$
\begin{aligned}
& (\tan \alpha)_{g r}-(\tan \alpha)_{i r r}=\frac{k Q_{T}}{\sqrt{2}}\left(\frac{L_{q}}{L_{T}}\right)^{\frac{1}{2}} \frac{I_{Z}}{I_{C}} \rho^{(e q)} \\
& \times \frac{T_{1} T_{2} \Omega_{1}^{2}}{1+\delta^{2} T_{2}^{2}+T_{1} T_{2} \Omega_{1}^{2}},
\end{aligned}
$$

which allows for the direct measurement from its width of the dephasing time $T_{2} 39$.

This technique is similar to that used in low field NMR, where a longitudinal magnetization can be measured directly with the aid of SQUID (see Ref. 40 and references therein).

In conclusion, we developed here a quantum theory for the calculation of the low frequency linear susceptibility of the interferometer type superconducting qubits. The obtained general results are applied for the calculation of the tank detuning and friction both for flux and charge qubit. For irradiated flux qubit we obtained explicit expression for the tank quality factor which allows us to calculate the recently found effect of the amplification and the damping of the tank. We have also shown the application of the theory to the continuous radio-frequency monitoring of the qubit with the aid of DC SQUID in the inductive mode. Our theory shows that radio-frequency method can also be applied for the investigation of other types of quantum two level structures provided the interaction between the tank and quantum system is weak.

\section{Acknowledgments}

Ya. S. G. thanks M. Grajcar and S. Shevchenko for fruitful discussions. This work was supported by the DFG under grant IL 150/1-1 and partly by the ESF under AQDJJ programme. E.I. thanks also the EU for support through the RSFQubit and the EuroSQIP projects.
1 Yu. Makhlin, G. Schön, and A. Shnirman, Rev. Mod. Phys. 73, 357 (2001).

${ }^{2}$ G. Wendin and V. S. Shumeiko, in Handbook of Theoretical and Computational Nanotechnology, edited by M. Rieth and W. Schommers (American Scientific Publishers, 2006), vol. 3 .

3 Caspar H. van der Wal, A. C. J. ter Haar, F. K. Wilhelm, R. N. Schouten, C. J. P. M. Harmans, T.P.Orlando, Seth Lloyd, and J. E. Mooij, Science 290, 773 (2000).

4 A. O. Niskanen, K. Harrabi, F. Yoshihara, Y. Nakamura, S. Lloyd, and J. S. Tsai, Science 316, 723 (2007).

5 Ya. S. Greenberg, A. Izmalkov, M. Grajcar, E. Ilichev, W. Krech, H.-G. Meyer, M.H.S. Amin, and A. Maassen van den Brink, Phys. Rev. B 66, 214525 (2002).

6 Ya. S. Greenberg, A. Izmalkov, M. Grajcar, E. Ilichev, W. Krech, and H.-G. Meyer, Phys. Rev. B 66, 224511 (2002).

7 M. Grajcar, A. Izmalkov, E. Ilichev, Th. Wagner, N. Oukhanski, U. Hübner, T. May, I. Zhilyaev, H.E. Hoenig, Ya.S. Greenberg, V.I. Shnyrkov, D. Born, W. Krech, H.G. Meyer, A. Maassen van den Brink, and M.H.S. Amin, Phys. Rev. B 69, 060501(R) (2004).

8 A. Wallraff, D. I. Schuster, A. Blais, L. Frunzio, R.-S. Huang, J. Majer, S. Kumar, S. M. Girvin and R. J. Schoelkopf, Nature (London) 431, 162 (2004).

9 A. Blais, J. Gambetta, A. Wallraff, D. I. Schuster, S. M. Girvin, M. H. Devoret, and R. J. Schoelkopf, Phys. Rev. A75, 032329 (2007).

10 A.B. Zorin, Physica C 368, 284 (2002).

11 W. Krech, M. Grajcar, D. Born, I. Zhilyaev, Th. Wagner, E. Il'ichev, Ya. Greenberg, Phys. Lett. A 303, 352 (2002).

12 D. Born, V.I. Shnyrkov, W. Krech, Th. Wagner, E. Ilichev, M. Grajcar, U. Hübner, and H.-G. Meyer, Phys. Rev. B
70, 180501(R) (2004).

13 V.I. Shnyrkov, Th. Wagner, D. Born, S.N. Shevchenko, W. Krech, A.N. Omelyanchouk, E. Ilichev, and H.-G. Meyer, Phys. Rev. B 73, 024506 (2006).

14 H. Zangerle, J. Könemann, B. Mackrodt, R. Dolata, S. V. Lotkhov, S. A. Bogoslovsky, M. Götz, and A.B. Zorin, Phys. Rev. B 73, 224527 (2006).

15 A. Lupascu, C. J. M. Verwijs, R. N. Schouten, C. J. P. M. Harmans, and J. E. Mooij, Phys. Rev. Lett. 93, 177006 (2004).

16 A. Lupascu, E. F. C. Driessen, L. Roschier, C. J. P. M. Harmans, and J. E. Mooij, Phys. Rev. Lett. 96, 127003 (2006).

17 I. Siddiqi, R. Vijay, M. Metcalfe, E. Boaknin, L. Frunzio, R. J. Schoelkopf, and M. H. Devoret, Phys. Rev. B 73, 054510 (2006).

18 S.H.W. van der Ploeg, A. Izmalkov, A. Maassen van den Brink, U. Hübner, M. Grajcar, E. Ilichev, H.-G. Meyer, and A.M. Zagoskin, Phys. Rev. Lett. 98, 057004 (2007).

19 A. Izmalkov, M. Grajcar, S. H. W. van der Ploeg, U. Hübner, E. Ilichev, H.-G. Meyer, and A. M. Zagoskin, Europhys. Lett. 76, 533 (2006).

20 M. Grajcar, A. Izmalkov, S. H. W. van der Ploeg, S. Linzen, T. Plecenik, Th. Wagner, U. Hübner, E. Ilichev, H.-G. Meyer, A. Yu. Smirnov, P. J. Love, A. Maassen van den Brink, M. H. S. Amin, S. Uchaikin, and A. M. Zagoskin, Phys. Rev. Lett. 96, 047006 (2006).

21 M. Grajcar, A. Izmalkov, S.H.W. van der Ploeg, S. Linzen, E. Ilichev, Th. Wagner, U. Hübner, H.-G. Meyer, A. Maassen van den Brink, S. Uchaikin, and A.M. Zagoskin, Phys. Rev. B 72, 020503(R) (2005).

22 E. Ilichev, A.Yu. Smirnov, M. Grajcar, A. Izmalkov, D. 
Born, N. Oukhanski, Th. Wagner, W. Krech, H.-G. Meyer, and A. Zagoskin, Fizika Nizkikh Temperatur, 30, 823 (2004).

23 This is different from the case of qubit embedded in a resonant microwave circuit, where the resonator together with a qubit are treated quantum mechanically ${ }^{9}$.

${ }^{24}$ C. Coen-Tannoudji, J. Dupont-Rock, and G. Grynberg, Atom-Photon Interactions. Basic Prinsiples and Applications. (John Wiley, New York, 1998).

25 M. Grajcar, S.H.W. van der Ploeg, A. Izmalkov, E. Ilichev, H.-G. Meyer, A. Fedorov, A. Shnirman, and Gerd Schön, arXiv:cond-mat/0708.0665v1 (unpublished).

26 Ya. S. Greenberg and W. Krech, arXiv:cond-mat/0601644 (unpublished).

27 Ya. S. Greenberg and E. Ilichev, arXiv:quant-ph/0502187 (unpublished).

28 Ya. S. Greenberg, E. Ilichev, and A. Izmalkov, Europhys. Lett., 72, 880 (2005)

29 Ya. S. Greenberg, Phys. Rev. B 76, 104520 (2007).

30 F. Bloch, Phys. Rev. 105, 1206 (1957).

31 A. G. Redfield, IBM J. Res. Dev. 1, 19 (1957).

${ }^{32}$ It is true that if the system is subjected to a strong external driving the $\Gamma$ 's rates and the corresponding rate equations can be substantially modified ${ }^{35,36}$. However, even in this case the most of experiments can adequately be explained by the Bloch equations with phenomenological $\Gamma$ 's rates as fitting parameters (see, for example, ${ }^{37}$ ).

${ }^{33}$ Frequently, the term "Rabi frequency" is associated with the quantity $\Omega_{1}$. Here we call by this term the quantity $\Omega_{R}$, the frequency with which the population oscillates if the high frequency detuning $\delta$ is different from zero.

${ }^{34}$ F. Bloch, Phys. Rev. 70, 460 (1946).

35 A. Yu. Smirnov, Phys. Rev. B 68, 134514 (2003).

${ }^{36}$ L. Hartmann, I. Goychuk, M. Grifoni, and P. Hanggi, Phys. Rev. E 61, R4687 (2000).

37 S. Saito, M. Thorwart, H. Tanaka, M. Ueda, H. Nakano, K. Semba, and H. Takayanagi, Phys. Rev. Lett. 93, 037001 (2004).

38 A. Lupascu, C. J. P. M. Harmans, and J. E. Mooij , Phys. Rev. B 71, 184506 (2005).

39 This curve can also be measured from the distribution of the switching currents with the help of underdamped DC SQUID ${ }^{3}$.

40 Ya. S. Greenberg, Rev. Mod. Phys. 70, 175 (1998). 